\title{
Automatic growth series for right-angled Coxeter groups
}

\author{
Rebecca Glover and Richard Scott \\ (Communicated by Vadim Ponomarenko)
}

\begin{abstract}
Right-angled Coxeter groups have a natural automatic structure induced by their action on a CAT(0) cube complex. The normal form for this structure is defined with respect to the generating set consisting of all cliques in the defining graph for the group. In this paper we study the growth series for right-angled Coxeter groups with respect to this automatic generating set. In particular, we show that there exist nonisomorphic Coxeter groups with the same automatic growth series, and give a comparison with the usual growth series defined with respect to the standard generating set.
\end{abstract}

\section{Introduction}

Given a group and a generating set the corresponding growth series is the power series whose coefficients are the numbers of group elements of a given length (measured with respect to the generating set). If elements of the group have a suitable normal form with respect to the generating set, then the growth series can be computed as a rational function. A classical example is the case of Coxeter groups. A Coxeter group $W$ is by definition a group given by a certain type of presentation, hence comes equipped with a set of generators, usually denoted by $S$. Growth series for Coxeter groups with respect to this standard generating set are known to be rational and are characterized by a simple inductive formula due to Steinberg [1968].

A Coxeter group is right-angled if any two generators either commute or generate an infinite dihedral group. Right-angled Coxeter groups are particularly nice to work with because they are completely characterized by the graph $\Gamma$ consisting of a vertex for each generator and an edge for each pair of commuting generators.

MSC2000: 05A15, 20F10, 20F55.

Keywords: Coxeter groups, growth series.

Glover was supported by the Pennello Fund of the Department of Mathematics and Computer Science at Santa Clara University. 
In this case, Steinberg's formula reduces to a simple expression involving only the numbers of cliques (complete subgraphs) in $\Gamma$ of each size.

Any right-angled Coxeter group admits a natural action on a CAT( 0$)$ cube complex [Davis 2002; 2008], hence by a result of Niblo and Reeves [1998] it acquires an induced automatic structure. In particular, this structure includes a normal form that is recognized by a finite state automaton (see [Epstein et al. 1992] for details). Although Coxeter groups were already known to be automatic, the automatic structure coming from the action on the Davis complex is with respect to a different generating set than $S$. The relevant generating set, which we call the automatic generating set, consists of a single generator for each clique in $\Gamma$. More precisely, the generator corresponding to a clique $\sigma$ is the product of the vertices of $\sigma$.

The purpose of this paper is to study the growth series of right-angled Coxeter groups with respect to this automatic generating set. In Section 2, we review relevant properties of right-angled Coxeter groups. In Section 3, we introduce a multivariate growth series that specializes (with suitable substitutions of variables) to either the standard growth series or the automatic growth series. In Section 4, we describe a procedure for computing this multivariate growth series, proving as a corollary that it is also a rational function. Although the automatic growth series is an invariant of the graph $\Gamma$, there does not seem to be any simple formula analogous to the formula of Steinberg. In particular, the growth series definitely depends on more than the numbers of cliques of each size. In Section 5, however, we impose a strong form of regularity on the graph $\Gamma$, and show that under this restriction the growth series does indeed depend only on the clique numbers. We use this fact to construct examples of nonisomorphic groups with the same automatic (and standard) growth series. Finally, in Section 6, we compare the standard and automatic growth rates of a right-angled Coxeter group. In general, one expects that enlarging the generating set will increase the growth rate. We prove this directly by proving the stronger result that if $W$ is infinite, then the sphere of radius $r$ is always smaller with respect to the standard generators than with respect to the automatic generators.

\section{Right-angled Coxeter groups}

Let $\Gamma$ be a simplicial graph with vertex set $S$ and edge set $E$. The right-angled Coxeter group (RACG) determined by $\Gamma$ is the group defined by the presentation

$$
\left.W=\langle s \in S| s^{2}=1 \text { for all } s \in S \text {, and }\left(s s^{\prime}\right)^{2}=1 \text { for all }\left\{s, s^{\prime}\right\} \in E\right\rangle .
$$

In other words, $W$ has an (involutive) generator for each vertex of $\Gamma$, and two generators commute whenever the corresponding vertices are joined by an edge in $\Gamma$. The flag completion of $\Gamma$ (also known as the nerve of $W$ ) is the largest 
simplicial complex $K$ on the vertex set $S$ such that $\Gamma$ coincides with the 1-skeleton of $K$. Thus, $K$ consists of all subsets $\left\{s_{1}, \ldots, s_{k}\right\} \subset S$ such that $\left\{s_{i}, s_{j}\right\} \in E$ for all $1 \leq i<j \leq k$. The following proposition will allow us to work with any one of $\Gamma$, $W$, or $K$ interchangeably.

Proposition 1. Let $W$ and $W^{\prime}$ be the RACGs associated with $\Gamma$ and $\Gamma^{\prime}$, respectively, and let $K, K^{\prime}$ be the corresponding nerves. Then

$$
W \cong W^{\prime} \Longleftrightarrow \Gamma \cong \Gamma^{\prime} \Longleftrightarrow K \cong K^{\prime} .
$$

Proof. The only implication that is not immediate is that isomorphic RACGs must come from isomorphic graphs. This is a theorem of Radcliffe [2001].

The length of an element $w \in W$ is the minimal $k$ such that $w=s_{1} \cdots s_{k}$ for $s_{i} \in S$. More generally, for any generating set $R \subset W$, we define the $R$-length of $w \in W$ to be the minimal $k$ such that $w=r_{1} \cdots r_{k}$ for $r_{i} \in R$. We let $l_{R}(w)$ denote the $R$-length of $w \in W$. Our primary interest in this paper is the generating set defined as follows. For any simplex $\sigma \in K$, let $w_{\sigma}=\prod_{s \in \sigma} s$. The element $w_{\sigma}$ is well-defined since any pair of vertices in $\sigma$ are joined by an edge, so the corresponding generators in $W$ commute. By convention, we let $w_{\varnothing}=1$. We let $K_{>\varnothing}$ denote the set of nonempty simplices in $K$, and we let $A$ denote the set $A=\left\{w_{\sigma} \in W \mid \sigma \in K_{>\varnothing}\right\}$. Note that since $w_{\{s\}}=s$ for all $s \in S, A$ is also a generating set for $W$. To distinguish between the two generating sets $S$ and $A$, we shall call the first the standard generating set and the second the automatic generating set.

Definition 2. The standard growth series and the automatic growth series for $W$ are the power series $W_{S}(t)$ and $W_{A}(t)$ defined by

$$
W_{S}(t)=\sum_{w \in W} t^{l_{S}(w)}, \quad W_{A}(t)=\sum_{w \in W} t^{l_{A}(w)} .
$$

The standard growth series for arbitrary Coxeter groups is known to be a rational function [Steinberg 1968]. In the right-angled case, this rational function has a particularly simple form in terms of the combinatorics of the simplicial complex $K$. Recall that the $f$-polynomial of $K$ is the generating function for the numbers of simplices; that is, $f(t)=1+f_{0} t+f_{1} t^{2}+\cdots$ where $f_{i}$ is the number of $i$ dimensional simplices in $K$. (Note that if $K$ is the flag completion of a graph $\Gamma$, then $f_{i}$ is the number of $(i+1)$-cliques in $\Gamma$.) The following formula can be found, for example, in [Davis 2008, Proposition 17.4.2].

Proposition 3. Let $W$ be a $R A C G$, and let $f(t)$ be the f-polynomial of the nerve. Then the standard growth series is given by the formula

$$
\frac{1}{W_{S}(t)}=f\left(\frac{-t}{1+t}\right) \text {. }
$$


This formula shows that, as an invariant for RACGs, the standard growth series is fairly coarse: it is easy to construct examples of nonisomorphic groups with the same standard growth.

Example 4. Let $K(=\Gamma)$ be a tree with $n$ vertices. The $f$-polynomial of $K$ is $f(t)=1+n t+(n-1) t^{2}$, so using the formula above, one obtains the standard growth series

$$
W_{S}(t)=\frac{(1+t)^{2}}{1+(2-n) t}
$$

for the corresponding Coxeter group. In particular, any two trees with the same number of vertices yield RACGs with the same standard growth series.

The purpose of this paper is to study the automatic growth series $W_{A}(t)$, which appears to be a much more subtle invariant of the group than $W_{S}(t)$.

\section{The total growth series}

Both the automatic and standard growth series for a RACG $W$ can be regarded as specializations of a certain multivariable growth series. This "total" growth series is defined in terms of a certain regular language associated to any simplicial complex $K$. In the case where $K$ is a flag complex (that is, $K$ is the flag completion of its 1 -skeleton), this regular language determines a normal form for the corresponding RACG.

Let $K$ be a simplicial complex and let $\mathscr{A}=K_{>\varnothing}$. For any $\sigma \in K$, we let $\operatorname{St}(\sigma)$ denote the (vertices of the) closed star of $\sigma$. That is,

$$
\operatorname{St}(\sigma)=\{s \in K \mid\{s\} \cup \sigma \in K\} .
$$

The regular language we have in mind for $K$ consists of certain words over the alphabet $\mathscr{A}$. Let $\mathscr{A}^{*}$ denote the free monoid on $\mathscr{A}$. We shall say that a word $\omega=\sigma_{1} \cdots \sigma_{n} \in \mathscr{A}^{*}$ is right-greedy if $\operatorname{St}\left(\sigma_{i+1}\right) \cap \sigma_{i}=\varnothing$ for all $1 \leq i<n$. We then let $\mathscr{L}_{K} \subset \mathscr{A}^{*}$ denote the language consisting of all right-greedy words.

Remark 5. It is not hard to see that $\mathscr{L}_{K}$ is in fact a regular language (see, for example, [Epstein et al. 1992], for a definition). We take as our states the set $\mathscr{S}=\mathscr{A} \cup\{\varnothing, \rho\}$ where $\varnothing$ is the start state, and $\rho$ is a single fail state (thus, the set of accept states is $\mathscr{Y}=\mathscr{A} \cup\{\varnothing\})$. We define the transition function $\mu: \mathscr{Y} \times \mathscr{A} \rightarrow \mathscr{Y}$ by

- $\mu(\tau, \sigma)=\sigma$ whenever $\tau \in \mathscr{Y}$ and $\operatorname{St}(\sigma) \cap \tau=\varnothing$, and

- $\mu(\tau, \sigma)=\rho$ otherwise.

It follows easily that $\mathscr{L}_{K}$ is the accepted language of the automaton $(\mathscr{Y}, \mathcal{A}, \mu, \mathcal{Y}, \varnothing)$. 
For any word $\omega \in \mathscr{A}^{*}$ and $\sigma \in \mathscr{A}$ we let $n_{\sigma}(\omega)$ denote the number of occurrences of $\sigma$ in $\omega$. Let $t$ be an $\mathscr{A}$-tuple $\left(t_{\sigma}\right)_{\sigma \in \mathscr{A}}$ of indeterminates, and for each word $\omega$, we let $t^{\omega}$ be the monomial

$$
t^{\omega}=\prod_{\sigma \in \mathscr{A}} t_{\sigma}^{n_{\sigma}(\omega)}
$$

In particular, $t^{\varnothing}=1$.

Definition 6. Let $K$ be a simplicial complex and let $\mathscr{L}_{K}$ be the corresponding rightgreedy language. Then the total growth series of $\mathscr{L}_{K}$ is the generating function

$$
\mathscr{L}_{K}(\boldsymbol{t})=\sum_{\omega \in \mathscr{L}_{K}} t^{\omega}
$$

In the event that $K$ is the nerve of a right-angled Coxeter group $W$ (that is, whenever $K$ is a flag complex), we let $\mathscr{L}_{W}$ denote the language $\mathscr{L}_{K}$. Note that in this case $\sigma \mapsto w_{\sigma}$ defines a bijection from $\mathscr{A}$ to the automatic generating set $A \subset W$, and hence induces a surjection $\mathscr{A}^{*} \rightarrow W$. We let $\pi: \mathscr{L}_{W} \rightarrow W$ denote the restriction of this map to the set of right-greedy words. More precisely, if $\omega=\sigma_{1} \cdots \sigma_{n} \in \mathscr{L}_{W}$, then $\pi(\omega)=w_{\sigma_{1}} \cdots w_{\sigma_{n}} \in W$. The following proposition says that $\mathscr{L}_{W}$ gives a normal form for elements of $W$. We omit the proof, which follows directly from Tits' solution to the word problem for Coxeter groups [Tits 1969].

Proposition 7. Let $W$ be a RACG. Then

(i) The map $\pi: \mathscr{L}_{W} \rightarrow W$ is a bijection.

(ii) For $\omega=\sigma_{1} \cdots \sigma_{n} \in \mathscr{L}_{W}$, the A-length of $\pi(\omega)$ is $n$.

(iii) For $\omega=\sigma_{1} \cdots \sigma_{n} \in \mathscr{L}_{W}$, the S-length of $\pi(\omega)$ is $l_{S}\left(w_{\sigma_{1}}\right)+\cdots+l_{S}\left(w_{\sigma_{n}}\right)$.

It follows from Proposition 7 that both the standard and automatic growth series for $W$ are specializations of the total growth series for $\mathscr{L}_{W}$.

Corollary 8. Let $W$ be a RACG. Then

(i) $W_{S}(t)=\mathscr{L}_{W}(\boldsymbol{t})$ after the substitutions $t_{\sigma}=t^{|\sigma|}, \sigma \in \mathscr{A}$, and

(ii) $W_{A}(t)=\mathscr{L}_{W}(t)$ after the substitutions $t_{\sigma}=t, \sigma \in \mathscr{A}$.

In light of this corollary, it makes sense to call the total growth series of the language $\mathscr{L}_{W}$ the total growth series of $W$.

\section{Calculating the total growth series}

Let $K$ be an arbitrary finite simplicial complex. In this section we describe a method for computing the total growth series of the right-greedy language $\mathscr{L}_{K}$. As a corollary we obtain that the total growth series is a rational function.

Let $\mathscr{A}=K_{>\varnothing}$. We let $\mathbb{C}[\mathscr{A}]$ denote the polynomial ring in the indeterminates $t_{\sigma}$, $\sigma \in \mathscr{A}$, and let $\mathbb{C}(\mathscr{A})$ denote the quotient field of rational functions. Similarly, we 
let $\mathbb{C} \llbracket \mathscr{A} \rrbracket$ denote the ring of formal power series and $\mathbb{C}((\mathscr{A}))$ denote the quotient field. Note that $\mathbb{C}(\mathscr{A})$ is a subfield of $\mathbb{C}((\mathscr{A}))$.

We define a transition matrix $M: K \times K \rightarrow \mathbb{C}[\mathscr{A}]$ for $\mathscr{L}_{K}$ as follows:

(1) If $\sigma=\tau=\varnothing$, then $M(\sigma, \tau)=1$.

(2) If $\operatorname{St}(\sigma) \cap \tau=\varnothing$, then $M(\sigma, \tau)=t_{\sigma}$.

(3) Otherwise, $M(\sigma, \tau)=0$.

We let $\mathbb{C}(\mathscr{A})^{K}$ (respectively $\left.\mathbb{C}((\mathscr{A}))^{K}\right)$ denote the vector space over $\mathbb{C}(\mathscr{A})$ (resp., $\mathbb{C}((\mathscr{A})))$ with standard basis $\left\{\boldsymbol{e}_{\sigma} \mid \sigma \in K\right\}$, and we regard $M$ as a $(K \times K)$-matrix over $\mathbb{C}(\mathscr{A})$ (resp., over $\mathbb{C}((\mathscr{A})))$. Our goal in this section is to prove the following.

Theorem 9. Let $\mathscr{L}=\mathscr{L}_{K}$ be the right-greedy language over the simplicial complex $K$, and let $M$ be the transition matrix.

(i) The $\lambda=1$ eigenspace of $M$ is 1-dimensional over $\mathbb{C}(\mathscr{A})$, and therefore also over $\mathbb{C}((\mathscr{A}))$.

(ii) If $\boldsymbol{v}(\boldsymbol{t})=\left(v_{\sigma}(\boldsymbol{t})\right)_{\sigma \in K}$ is an eigenvector in $\mathbb{C}((\mathscr{A}))^{K}$ corresponding to the eigenvalue 1 , then $v_{\varnothing}(t) \neq 0$ and the total growth series for $\mathscr{L}_{K}$ is given by

$$
\mathscr{L}_{K}(\boldsymbol{t})=\sum_{\sigma \in K} \frac{v_{\sigma}(\boldsymbol{t})}{v_{\varnothing}(\boldsymbol{t})} .
$$

In particular, the total growth series is a rational function which can be computed explicitly via Gaussian elimination on $M$.

Proof. Since $M(\varnothing, \varnothing)=1$ and $M(\varnothing, r)=0$ for $r \neq \varnothing$, the $\varnothing$-row of the matrix $M-1$ is all zeros. Thus, to prove the first part of the theorem, it suffices to show that the $(K-\varnothing) \times(K-\varnothing)$ minor of $M-1$ is nonsingular (over $\mathbb{C}(\mathscr{A})$ ). This submatrix has polynomial entries whose constant terms are all zero off the diagonal and are all -1 on the diagonal. It follows that the determinant of this submatrix must be a polynomial which evaluates to \pm 1 when all of the indeterminates are zero, hence the determinant is nonzero in $\mathbb{C}(\mathscr{A})$. This proves that the $\lambda=1$ eigenspace of $M$ is 1-dimensional and that any eigenvector $\boldsymbol{v}(\boldsymbol{t})$ must have $v_{\varnothing}(\boldsymbol{t}) \neq 0$.

For the second part of the theorem, suppose $\boldsymbol{v}(\boldsymbol{t}) \in \mathbb{C}((\mathscr{A}))^{K}$ is any $\lambda=1$ eigenvector. For each $\sigma \in K$, let $\mathscr{L}_{\sigma}$ denote the set of words in $\mathscr{L}$ ending in $\sigma$, and let $u_{\sigma}(t)$ be the total growth series for $\mathscr{L}_{\sigma}$, that is,

$$
u_{\sigma}(\boldsymbol{t})=\sum_{\omega \in \mathscr{L}_{\sigma}} t^{\omega} .
$$

Then

$$
\mathscr{L}_{K}(\boldsymbol{t})=\sum_{\sigma \in K} u_{\sigma}(\boldsymbol{t}),
$$


and since only the trivial word ends in $\varnothing$, we have $u_{\varnothing}(t)=1$. In light of the first part of the theorem, it suffices to show that the vector $\boldsymbol{u}(\boldsymbol{t})=\left(u_{\sigma}(\boldsymbol{t})\right)_{\sigma \in K} \in \mathbb{C}((\mathscr{A}))^{K}$ is also an eigenvector for $M$ with eigenvalue 1 (since this will then imply that $u_{\sigma}(\boldsymbol{t})=v_{\sigma}(\boldsymbol{t}) / v_{\varnothing}(\boldsymbol{t})$. $)$

For any integer $n \geq 0$ we let $\mathscr{L}_{\sigma}^{(n)}$ be the subset of $\mathscr{L}_{\sigma}$ consisting of words of length $\leq n$, and let $\boldsymbol{u}^{(n)}(\boldsymbol{t})=\left(u_{\sigma}^{(n)}(\boldsymbol{t})\right)_{\sigma \in K}$ where $u_{\sigma}^{(n)}(\boldsymbol{t})$ is the growth series for $\mathscr{L}_{\sigma}^{(n)}$ (a polynomial of degree $n$ ). In particular, if we regard $\boldsymbol{u}(\boldsymbol{t})$ as a power series with vector coefficients, then $\boldsymbol{u}^{(n)}(\boldsymbol{t})$ is the $n$th partial sum. Now any word $\omega \in \mathscr{L}_{\tau}^{(n)}$ can be extended to a word $\omega \sigma \in \mathscr{L}_{\sigma}^{(n+1)}$ precisely when $\operatorname{St}(\sigma) \cap \tau=\varnothing$, and in this case $t^{\omega \sigma}=t^{\omega} \cdot t_{\sigma}$. It then follows from the definition of $M$ that

$$
M \boldsymbol{u}^{(n)}(\boldsymbol{t})=\boldsymbol{u}^{(n+1)}(\boldsymbol{t})
$$

for all $n \geq 0$. But since $\boldsymbol{u}^{(n)}(\boldsymbol{t})$ is the $n$th partial sum of $\boldsymbol{u}(\boldsymbol{t})$, this means that $\boldsymbol{u}(\boldsymbol{t})$ must be a $\lambda=1$ eigenvector of $M$.

Corollary 10. Let $W$ be a $R A C G$. Then the total growth series $\mathscr{L}_{W}(t)$ is a rational function in the indeterminates $t_{\sigma}, \sigma \in K_{>\varnothing}$; thus (by Corollary 8) the standard and automatic growth series for $W$ are rational functions of the single indeterminate $t$.

More importantly, Theorem 9 describes exactly how to obtain these power series.

Example 11. Consider the Coxeter groups $W$ and $W^{\prime}$ corresponding to these trees:
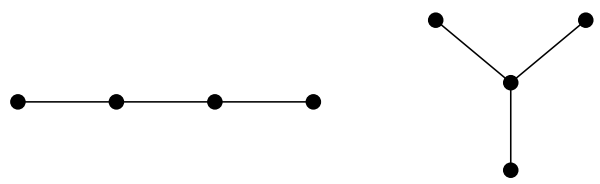

The matrix $M$ for $W$ (with respect to the ordering $\varnothing, 1,2,3,4,12,23,34$ ) is

$$
M=\left[\begin{array}{cccccccc}
1 & 0 & 0 & 0 & 0 & 0 & 0 & 0 \\
t_{1} & 0 & 0 & t_{1} & t_{1} & 0 & 0 & t_{1} \\
t_{2} & 0 & 0 & 0 & t_{2} & 0 & 0 & 0 \\
t_{3} & t_{3} & 0 & 0 & 0 & 0 & 0 & 0 \\
t_{4} & t_{4} & t_{4} & 0 & 0 & t_{4} & 0 & 0 \\
t_{12} & 0 & 0 & t_{12} & t_{12} & 0 & 0 & t_{12} \\
t_{23} & t_{23} & 0 & 0 & t_{23} & 0 & 0 & 0 \\
t_{34} & t_{34} & t_{34} & 0 & 0 & t_{34} & 0 & 0
\end{array}\right]
$$

We find the total growth series by finding any vector in the nullspace of $M-1$, dividing by the first entry of that vector, and then adding up the entries of the 
vector:

$$
\mathscr{L}(\boldsymbol{t})=\frac{\left(\begin{array}{c}
1+t_{34} t_{12}+t_{2}+t_{3}+t_{4}+t_{1}+t_{23}+t_{12}+t_{34} \\
-t_{3} t_{4} t_{2}+t_{12} t_{3}+t_{4} t_{2}+t_{23} t_{4}+t_{34} t_{1}+t_{34} t_{2}+t_{4} t_{12}+t_{1} t_{3}+t_{4} t_{1} \\
-t_{1} t_{3} t_{2}-t_{1} t_{3} t_{4} t_{2}-t_{23} t_{34} t_{12}+t_{23} t_{2} t_{34} t_{1}+t_{23} t_{12} t_{3} t_{4} \\
+t_{23} t_{1}+t_{23} t_{4} t_{1}-t_{23} t_{1} t_{3} t_{4} t_{2}
\end{array}\right)}{1-t_{4} t_{12}-t_{34} t_{1}-t_{1} t_{3}-t_{34} t_{12}+t_{1} t_{3} t_{4} t_{2}-t_{4} t_{2}-t_{4} t_{1}} .
$$

By making the substitutions in Corollary 8 , we get the automatic growth series

$$
W_{A}(t)=\frac{1+5 t+t^{2}+t^{3}}{1-2 t-t^{2}} .
$$

By performing the same steps for $W^{\prime}$ we find the automatic growth series

$$
W_{A^{\prime}}^{\prime}(t)=\frac{1+5 t-2 t^{2}}{1-2 t} .
$$

Thus, the automatic growth series can tell these groups apart, while the standard growth series cannot (see Example 4).

The example above shows that the automatic growth series is not determined by the $f$-polynomial (as is the standard growth series). In general, the properties of the complex $K$ that determine the automatic growth series seem to be fairly subtle. However, in the next section we describe a class of simplicial complexes for which the automatic growth series is still determined by the $f$-polynomial (but even in this case, the formula for the automatic growth series in terms of the $f$-polynomial is very complicated).

\section{Link-regular simplicial complexes}

In this section we consider certain simplicial complexes $K$ whose structure allows for a substantial reduction in the recursion defining the language $\mathscr{L}_{K}$. For this class of simplicial complexes, both the automatic growth series and the standard growth series are determined by the $f$-polynomial of $K$. We use this fact to obtain nonisomorphic Coxeter groups that have the same automatic growth series. To define our regularity condition, we first recall the that the link of a simplex $\sigma$ in $K$ is, by definition, the subcomplex of $K$ consisting of all $\tau \in K$ such that $\sigma \cup \tau \in K$ and $\sigma \cap \tau=\varnothing$.

Definition 12. Let $K$ be a finite flag simplicial complex of dimension $d$. We say that $K$ is link-regular if for every $0 \leq j \leq d$, the link of every $j$-simplex $\sigma \in K$ has the same $f$-polynomial. In this case, we let $F_{j}(t)$ denote the $f$-polynomial of the link of a $j$-simplex in $K$. (Since we regard $\operatorname{dim} \varnothing=-1$, the $f$-polynomial for $K$ itself is $F_{-1}(t)$.) 
Proposition 13. Let $K$ be a link-regular simplicial complex of dimension $d$. Then

$$
F_{j}(t)=\frac{f^{(j+1)}(t)}{(j+1) ! f_{j}}
$$

where $f(t)=1+f_{0} t+\cdots+f_{d} t^{d+1}$ is the f-polynomial for $K$ and $f^{(j+1)}(t)$ denotes its $(j+1)$ st derivative.

Proof. Let $f_{k}^{j}$ denote the number of $k$-simplices in the link of a $j$-simplex, so

$$
F_{j}(t)=1+f_{0}^{j} t+f_{1}^{j} t^{2}+\cdots+f_{d-j}^{j} t^{d-j+1} .
$$

Any $k$-simplex $\tau$ in the link of a $j$-simplex $\sigma$ determines a $(k+j+1)$-simplex $\sigma \cup \tau$ in $K$, and there are $\left(\begin{array}{c}k+j+1 \\ j+1\end{array}\right)$ such ways to obtain this simplex. This gives the relation

$$
f_{j} f_{j}^{k}=\left(\begin{array}{c}
k+j+1 \\
j+1
\end{array}\right) f_{k+j+1} .
$$

Solving for $f_{j}^{k}$ and substituting for the coefficients in the polynomial $F_{j}(t)$ gives the desired identity.

By Proposition 3, we know that two Coxeter groups will have the same standard growth series if their nerves have the same $f$-polynomial. If in addition, we assume their nerves are link-regular, we obtain the following theorem.

Theorem 14. Let $W$ and $W^{\prime}$ be two right-angled Coxeter groups with corresponding nerves $K$ and $K^{\prime}$. Assume further that $K$ and $K^{\prime}$ are both link-regular and have the same f-polynomial. Then $W$ and $W^{\prime}$ have the same automatic growth series.

Proof. Let $K$ be a link-regular simplicial complex of dimension $d$. By Proposition 13 , it suffices to show that the automatic growth series depends only on the polynomials $F_{j}(t),-1 \leq j \leq d$.

Let $\mathscr{L}=\mathscr{L}_{K}$, and for each $i \in \mathbb{Z}_{\geq 0}$, let $B_{i}(m)$ denote the number of words of length $m$ in $\mathscr{L}$ that end in an $i$-simplex. Then the automatic growth series can be written as the double sum

$$
W_{A}(t)=1+\sum_{m=1}^{\infty} \sum_{i=0}^{d} B_{i}(m) t^{m} .
$$

We form a recursion relation for $B_{i}(m), m \geq 2$, as follows. Any word $\omega$ of length $m$ that ends in an $i$-simplex is obtained by multiplying a word $\omega^{\prime}$ of length $m-1$ by some $i$-simplex $\sigma$. Given $\omega^{\prime} \in W$ of length $m-1$ ending in $\tau$, let $\beta_{i}(\tau)$ be the number of $i$-simplices $\sigma$ such that $\tau \sigma \in \mathscr{L}$. That is, $\beta_{i}(\tau)$ is the number of $i$-simplices $\sigma$ such that $S t(\sigma) \cap \tau=\varnothing$. Since $K$ is link-regular, $\beta_{i}(\tau)$ depends only on the dimension of $\tau$ and not on $\tau$ itself. We denote this number by $\beta_{i j}$ where 
$j=\operatorname{dim}(\tau)$. The number of words of length $m$ ending in an $i$-simplex is then given by the recurrence

$$
B_{i}(m)=\beta_{i 0} B_{0}(m-1)+\beta_{i 1} B_{1}(m-1)+\cdots+\beta_{i d} B_{d}(m-1)
$$

for $m \geq 2$. A straightforward inclusion-exclusion argument gives an explicit formula for $\beta_{i j}$ in terms of the $f$-polynomials of the various links; more explicitly, if one writes the polynomials of the links as

$$
F_{j}(t)=1+f_{0}^{j} t+f_{1}^{j} t^{2}+\cdots+f_{d-j}^{j} t^{d-j+1},
$$

then the $\beta_{i j}$ are given by

$$
\beta_{i j}=f_{i}+\sum_{q=1}^{j+1}(-1)^{q}\left(\begin{array}{c}
j+1 \\
q
\end{array}\right) \sum_{p=0}^{q}\left(\begin{array}{l}
q \\
p
\end{array}\right) f_{i-p}^{q-1} .
$$

Since the coefficients of the recurrence relations and the initial values $B_{i}(1)$ (which equals $f_{i}^{-1}=f_{i}$ ) can all be expressed explicitly in terms of the $f$-polynomials of the links, so can all of the $B_{i}(m)$ s. Thus $W_{A}(t)$ depends only on the $f$-polynomial of $K$.

We now give several pairs of examples of nonisomorphic RACGs that have the same automatic (and standard) growth series.

Example 15. The Coxeter groups corresponding to these two graphs have the same automatic growth series:
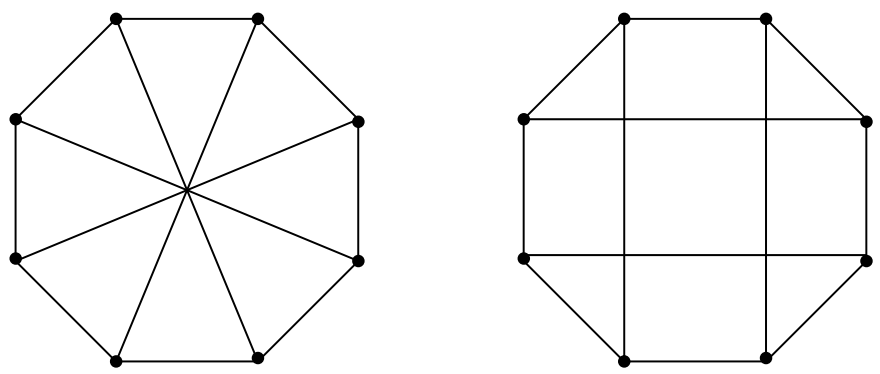

To see this, note that any vertex-regular graph with no 3-cycles is a 1-dimensional link-regular simplicial complex; hence these graphs are both link-regular and have the same $f$-polynomial $f(t)=1+8 t+12 t^{2}$. By Theorem 14, they have the same automatic growth series, which we can compute explicitly as

$$
W_{A}(t)=\frac{1+9 t+2 t^{2}}{1-11 t+10 t^{2}} .
$$

The graphs are clearly not isomorphic (the one on the right is bipartite, the other is not). 
It is easy to generalize the example above to other pairs of Coxeter groups with the same automatic growth series by adding diagonals to a $2 n$-gon to get a regular graph. As long as there are no 3-cycles, these graphs will have simplicial complexes that are link-regular. (In fact, one can construct such a pair $\Gamma_{h y p}$ and $\Gamma_{e u c}$ so that the first has no 4-cycles while the second has 4-cycles. The corresponding Coxeter groups will have the same standard and automatic growth series even though one is a Gromov hyperbolic group and the other is not.)

Constructing examples with $\operatorname{dim} K \geq 2$ is a little more subtle:

Example 16. Let $\Gamma_{1}$ and $\Gamma_{2}$ be the following two graphs, embedded on the torus and the Klein bottle, respectively:
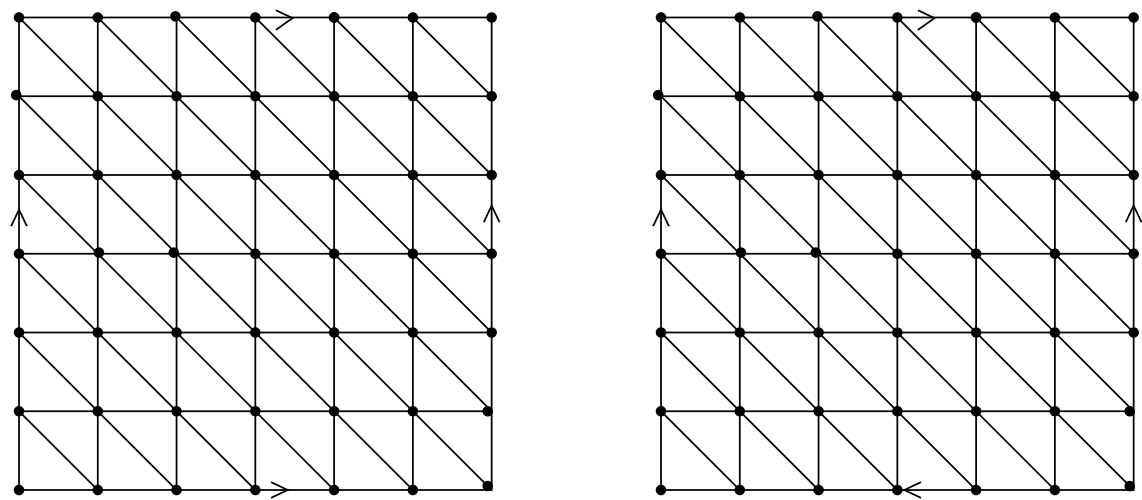

They are not isomorphic ( $\Gamma_{1}$ is three-colorable and $\Gamma_{2}$ is not), so they correspond to nonisomorphic Coxeter groups. On the other hand, the corresponding nerves are precisely the triangulations shown in the figure. These simplicial complexes are clearly link-regular and have the same $f$-polynomials, hence have the same automatic growth series.

We have seen examples of two nonisomorphic right-angled Coxeter groups where the standard growth series are the same, but the automatic growth series are different and where both the standard and the automatic growth series are the same. This suggests the following question, for which we do not yet have an answer.

Question 17. Are there two right-angled Coxeter groups with the same automatic growth series but different standard growth series?

\section{Comparing automatic and standard growth rates}

Given a power series $\sum a_{n} t^{n}$ (over $\mathbb{C}$ ), we define its growth rate $\gamma$ to be

$$
\gamma=\limsup _{n} \sqrt[n]{\left|a_{n}\right|}
$$


Equivalently, by the root test, $\gamma=1 / \rho$ where $\rho$ is the radius of convergence. If a power series is a rational function say $p(t) / q(t)$, its growth rate can therefore be calculated from

$$
\frac{1}{\gamma}=\rho=\min \left(\left|r_{1}\right|,\left|r_{2}\right|, \ldots,\left|r_{n}\right|\right),
$$

where $r_{1}, \ldots, r_{n}$ are the roots of the denominator $q(t)$.

In this section, we consider the growth rates for the standard and automatic growth series of a RACG. We denote them by $\gamma_{S}$ and $\gamma_{A}$, respectively. Our first observation is following.

Proposition 18. If $(W, S)$ is a right-angled Coxeter group, then $\gamma_{S} \leq \gamma_{A}$. More precisely:

(i) If $W$ is finite, $\gamma_{S}=\gamma_{A}=0$.

(ii) If $W$ is infinite and virtually abelian, $\gamma_{S}=\gamma_{A}=1$.

(iii) If $W$ is not virtually abelian, $1<\gamma_{S} \leq \gamma_{A}$.

Proof. Statement (i) is clear. Statement (ii) follows from the fact that for an arbitrary infinite Coxeter group $W$ and any generating set, the radius of convergence for the growth series is 1 if and only if $W$ is virtually abelian [Davis 2008, Proposition 17.2.1]. This also ensures that if $W$ is not virtually abelian, then the two growth rates $\gamma_{S}$ and $\gamma_{A}$ are both $>1$. For the final inequality in (iii), suppose $W$ is not virtually abelian. Let $B W_{S}(t)$ (respectively, $B W_{A}(t)$ ) denote the growth series for balls in $W$ with respect to the $S$-length (resp., $A$-length). In other words, $B W_{S}(t)=\sum_{n} b_{n} t^{n}$ where $b_{n}$ is the number of elements in $W$ of $S$-length $\leq n$. A geometric series calculation shows that

$$
B W_{S}(t)=\frac{W_{S}(t)}{1-t}, \quad B W_{A}(t)=\frac{W_{A}(t)}{1-t} .
$$

Since the growth rate of $W_{S}(t)$ (respectively, $\left.W_{A}(t)\right)$ is $>1, B W_{S}(t)$ (resp., $B W_{A}(t)$ ) will have the same growth rate as $W_{S}(t)$ (resp., $\left.W_{A}(t)\right)$. On the other hand since $S \subset A$, the $A$-length of an element is always $\leq$ the $S$-length, hence the terms of the series $B W_{A}(t)$ are all $\geq$ the terms of the series $B W_{S}(t)$. It follows that the growth rate for $B W_{S}(t)$ is $\leq$ the growth rate for $B W_{A}(t)$; in other words, $\gamma_{S} \leq \gamma_{A}$.

The following examples illustrate these three cases.

Example 19. Let $W$ be the Coxeter group whose graph $\Gamma$ is the complete graph $K_{n}$. Then $W$ is the (finite) group $\left(\mathbb{Z}_{2}\right)^{n}$ and the standard and automatic growth series are

$$
\begin{aligned}
& W_{S}(t)=(1+t)^{n}=1+n t+\cdots+n t^{n-1}+t^{n}, \\
& W_{A}(t)=1+\left(2^{n}-1\right) t .
\end{aligned}
$$

Since these are both polynomials, $\gamma_{S}=\gamma_{A}=0$. 
Example 20. Let $(W, S)$ be the Coxeter group corresponding to the graph $K_{4}-e$, the complete graph on 4 vertices with one edge removed. The standard growth series and automatic growth series are

$$
\begin{aligned}
& W_{S}(t)=\frac{(1+t)^{3}}{1-t}=1+4 t+7 t^{2}+8 t^{3}+8 t^{4} \ldots, \\
& W_{A}(t)=\frac{1+10 t-3 t^{2}}{1-t}=1+11 t+8 t^{2}+8 t^{3}+8 t^{4} \ldots
\end{aligned}
$$

Hence both have growth rates equal to 1 . In this case the group $W$ is a product of $\left(\mathbb{Z}_{2}\right)^{2}$ with the infinite dihedral group (hence is virtually $\mathbb{Z}$ ).

Example 21. Recall the Coxeter group $W$ from Example 11. The growth series are given by

$$
\begin{aligned}
W_{S}(t) & =\frac{(1+t)^{2}}{1-2 t}, \\
W_{A}(t) & =\frac{1+5 t+t^{2}+t^{3}}{1-2 t-t^{2}},
\end{aligned}
$$

so we obtain

$$
\gamma_{S}=2, \quad \gamma_{A}=\frac{1}{-1+\sqrt{2}} \approx 2.41 \text {. }
$$

It turns out that the inequality $\gamma_{S} \leq \gamma_{A}$ can also be deduced from a stronger statement about the relative growth rates of the coefficient sequences in $W_{S}(t)$ and $W_{A}(t)$. Namely, let $\mathscr{L}=\mathscr{L}_{W}$ be the language defining the right-greedy normal form for $W$, and let $S_{n}$ (respectively, $A_{n}$ ) denote the set of words in $\mathscr{L}$ with $S$-length $n$ (resp., $A$-length $n$ ). Then the relevant growth series are given by

$$
\begin{aligned}
& W_{S}(t)=1+\left|S_{1}\right| t+\left|S_{2}\right| t^{2}+\left|S_{3}\right| t^{3}+\cdots, \\
& W_{A}(t)=1+\left|A_{1}\right| t+\left|A_{2}\right| t^{2}+\left|A_{3}\right| t^{3}+\cdots .
\end{aligned}
$$

It is clear that $\gamma_{S} \leq \gamma_{A}$ would be implied by the stronger statement $\left|S_{n}\right| \leq\left|A_{n}\right|$ for all $n$. Of course, this statement is false if $K$ is a simplex (that is, if $W$ is finite) as in Example 19, but otherwise, we have the following.

Theorem 22. Let $(W, S)$ be an infinite right-angled Coxeter group. Then the coefficients of the standard and automatic growth series satisfy $\left|S_{n}\right| \leq\left|A_{n}\right|$ for all $n \geq 0$.

Before proving the theorem, we recall some terminology for simplicial complexes. The $m$-skeleton $K^{(m)}$ of a simplicial complex $K$ is the subcomplex consisting of all $\sigma \in K$ such that $\operatorname{dim}(\sigma) \leq m$. Thus, the vertex set of $K$ is denoted $K^{(0)}$ and when $K$ is the flag completion of a graph $\Gamma$, the 1 -skeleton $K^{(1)}$ is precisely the graph $\Gamma$. 
Given a simplex $\sigma \in K$, we let $\hat{\sigma}$ denote the subcomplex consisting of $\sigma$ and its faces. And given two simplicial complexes $K_{1}$ and $K_{2}$, we define their join $K_{1} * K_{2}$ to be the simplicial complex with vertex set $K_{1}^{(0)} \cup K_{2}^{(0)}$ and simplices given by

$$
K_{1} * K_{2}=\left\{\sigma_{1} \cup \sigma_{2} \mid \sigma_{1} \in K_{1} \text { and } \sigma_{2} \in K_{2}\right\} .
$$

Lemma 23. Let $K$ be a flag simplicial complex. Then there exists $a \sigma \in K$ and $a$ subcomplex $L \subseteq K$ such that $K=\hat{\sigma} * L$ and for any $\tau \in L, \operatorname{St}(\tau) \neq L^{(0)}$.

Proof. Let $I=\left\{\sigma_{1}, \sigma_{2}, \ldots, \sigma_{q}\right\}$ be the set of all $\sigma_{i}$ such that $\operatorname{St}\left(\sigma_{i}\right)=K^{(0)}$. Then all of the vertices of $\sigma_{i}$ are adjacent to all of the vertices of $\sigma_{j}$ for $1 \leq i \leq j \leq q$. Since $K$ is a flag complex this means that $\sigma=\sigma_{1} \cup \sigma_{2} \cup \ldots \cup \sigma_{q}$ is a simplex in $K$. Let $L$ be the induced subcomplex on the vertex set $K^{(0)}-\sigma$. Since $K$ is a flag complex and all of the vertices in $L$ are adjacent to all of the vertices in $\sigma$, we know that $\hat{\sigma} * L=K$. If there exists a $\tau \in L$ such that $\operatorname{St}(\tau)=L^{(0)}$, then in fact $\operatorname{St}(\tau)=K^{(0)}$ by the definition of the join, contradicting our definition of $I$. Hence, $L$ has the desired property.

Proof of Theorem 22. To show that $\left|S_{n}\right| \leq\left|A_{n}\right|$, it suffices to construct an injective map $S_{n} \rightarrow A_{n}$. By Lemma 23, we can write $K$ in the form $K=\hat{\sigma} * L$ where $L \neq\{\varnothing\}$ and for any $\tau \in L, \operatorname{St}(\tau) \neq L^{(0)}$. Let $\omega=\sigma_{1} \sigma_{2} \cdots \sigma_{p}$ be an element in $S_{n}$. Then for each $i, \sigma_{i}=\tau_{i} \cup \sigma_{i}^{\prime}$ for some $\tau_{i} \in \hat{\sigma}$ and $\sigma_{i}^{\prime} \in L$. If $\sigma_{i}^{\prime}=\varnothing$ for some $1<i \leq p, \operatorname{St}\left(\sigma_{i+1}\right) \cap \sigma_{i} \neq \varnothing$, contradicting the fact that $\omega \in \mathscr{L}$. Therefore, $\sigma_{i}^{\prime} \neq \varnothing$ for all $1<i \leq p$.

In order to map $\omega$ to an element of $A_{n}$, we will append $n-p 0$-simplices to the front of $\omega$ while keeping it in $\mathscr{L}$. There are two cases.

Case 1. $\sigma_{1}^{\prime} \neq \varnothing$. We know that $\operatorname{St}\left(\sigma_{1}^{\prime}\right) \neq L^{(0)}$ so $\operatorname{St}\left(\sigma_{1}\right) \neq K^{(0)}$. Therefore, there exists a $v \in L^{(0)}$ such that $\operatorname{St}\left(\sigma_{1}\right) \cap\{v\}=\varnothing$, this means that $\{v\} \omega$ is still in $\mathscr{L}$. Since $v \in L^{(0)}$, there exists a $u \in L^{(0)}$ such that $\operatorname{St}(\{v\}) \cap\{u\}=\varnothing$. It follows that the words, $\{v\} \omega,\{u\}\{v\} \omega,\{v\}\{u\}\{v\} \omega, \ldots$ are all in $\mathscr{L}$, so by adjoining the alternating word $v=\{u\}\{v\} \cdots\{v\}$ (or $v=\{v\}\{u\}\{v\} \cdots\{v\}$ depending on the parity of $n-p$ ) of length $(n-p)$ to the beginning of $\omega$, we obtain an element $v \omega \in A_{n}$.

Case 2. $\sigma_{1}^{\prime}=\varnothing$. In this case $\omega=\tau_{1} \sigma_{2} \cdots \sigma_{p}$. Since $\tau_{1} \in \hat{\sigma}$, this means that $\tau_{1} \subset \operatorname{St}\left(\sigma_{2}\right)$ contradicting the fact that $\omega \in \mathscr{L}$. It follows that in this case, we must have $p=1$ and $\omega=\tau_{1}$. Put $\tau=\tau_{1}$. Since $\omega \in S_{n}$ we know $|\tau|=n$. Every element in $\tau$ is adjacent to every vertex in $L$ so pick a $v \in L^{(0)}$. Then $\{v\} \cup \tau \in \mathscr{L}$. Moreover, $\operatorname{St}(\{v\} \cup \tau) \neq K^{(0)}$, so there exists a $u \in L^{(0)}$ such that $\operatorname{St}(\{v\} \cup \tau) \cap\{u\}=\varnothing$. In particular, $u$ and $v$ are not adjacent, so if we let $v$ be the alternating word $\{u\}\{v\} \cdots\{u\}$ (or $\{v\}\{u\}\{v\} \cdots\{u\}$ depending on the parity of $n$ ) of length $n-1$ to the beginning of $\{v\} \cup \tau$, we obtain an element $v(\{v\} \cup \tau) \in A_{n}$. 
Since two words in $\mathscr{L}$ with different endings must be different, the map $S_{n} \rightarrow A_{n}$ given by $\omega \mapsto v \omega$ (Case 1) or $\omega \mapsto v(\{v\} \cup \tau)$ (Case 2) is injective.

\section{References}

[Davis 2002] M. W. Davis, "Nonpositive curvature and reflection groups”, pp. 373-422 in Handbook of geometric topology, edited by R. J. Daverman and R. B. Sher, North-Holland, Amsterdam, 2002. MR 2002m:53061 Zbl 0998.57002

[Davis 2008] M. W. Davis, The geometry and topology of Coxeter groups, London Mathematical Society Monographs Series 32, Princeton University Press, Princeton, NJ, 2008. MR 2008k:20091 Zbl 1142.20020

[Epstein et al. 1992] D. B. A. Epstein, J. W. Cannon, D. F. Holt, S. V. F. Levy, M. S. Paterson, and W. P. Thurston, Word processing in groups, Jones and Bartlett, Boston, MA, 1992. MR 93i:20036 Zbl 0764.20017

[Niblo and Reeves 1998] G. A. Niblo and L. D. Reeves, "The geometry of cube complexes and the complexity of their fundamental groups", Topology 37:3 (1998), 621-633. MR 99a:20037 Zbl 0911.57002

[Radcliffe 2001] D. Radcliffe, Unique presentation of Coxeter groups and related groups, Ph.D. thesis, University of Wisconsin, Milwaukee, 2001. Ph.D. thesis.

[Steinberg 1968] R. Steinberg, Endomorphisms of linear algebraic groups, Memoirs of the American Mathematical Society 80, American Mathematical Society, Providence, 1968. MR 37 \#6288 Zbl 0164.02902

[Tits 1969] J. Tits, "Le problème des mots dans les groupes de Coxeter", pp. 175-185 in Symposia Mathematica (INDAM, Rome, 1967/68), vol. 1, Academic Press, London, 1969. MR 40 \#7339 Zbl 0206.03002

Received: 2008-03-11 Revised: 2009-09-08 Accepted: 2009-09-26

reglover@email.unc.edu Department of Mathematics, The University of North Carolina, CB No. 3250, Phillips Hall, Chapel Hill, NC 27599-3250, United States

rscott@schubert.scu.edu

Department of Mathematics and Computer Science,

Santa Clara University, 500 El Camino Real, Santa Clara, CA 95053-0290, United States http://schubert.scu.edu/rscott 